\title{
Abordagens de modelagem para investigar a interação entre Fulereno e $\beta$-Amiloide
}

\author{
Modeling approaches to investigate the interaction between Fullerene and $\beta$-Amyloid \\ Enfoques de modelado para investigar la interacción entre el Fullereno y el $\beta$-amiloide
}

Recebido: 19/08/2021 | Revisado: 23/08/2021 | Aceito: 28/08/2021 | Publicado: 05/09/2021

\author{
Felipe Magno da Cruz Junior \\ ORCID: https://orcid.org/0000-0003-1532-3104 \\ Universidade Federal do Pará, Brasil \\ E-mail: felipemag71@ hotmail.com \\ Jorddy Neves da Cruz \\ ORCID: https://orcid.org/0000-0003-0529-3714 \\ Universidade Federal do Pará, Brasil \\ E-mail: jorddynevescruz@gmail.com \\ Fabrine Silva Alves \\ ORCID: https://orcid.org/0000-0002-6602-1942 \\ Universidade Federal do Pará, Brasil \\ E-mail: fafa.bine@yahoo.com.br \\ Isaque Gemaque de Medeiros \\ ORCID: https://orcid.org/0000-0002-7974-8868 \\ Universidade Federal do Pará, Brasil \\ E-mail: igemaque@yahoo.com.br \\ Gleice Vasconcelos da Silva Pereira \\ ORCID: https://orcid.org/0000-0002-9702-250X \\ Universidade Federal do Pará, Brasil \\ E-mail: gleice_vasconcelos@hotmail.com \\ Luciano Augusto de Sousa \\ ORCID: https://orcid.org/0000-0002-7931-8307 \\ Secretaria de Estado de Educação, Brasil \\ E-mail: professorlucianoaugust@gmail.com \\ Adria Evellin Godinho de Vilhena \\ ORCID: https://orcid.org/0000-0002-9372-4993 \\ Universidade Federal do Pará, Brasil \\ E-mail: adriavilhena@gmail.com \\ Luciane do Socorro Nunes dos Santos Brasil \\ ORCID: https://orcid.org/0000-0001-5121-009X \\ Universidade Federal do Pará, Brasil \\ E-mail: 1snsbrasil@ig.com.br \\ José de Arimatéia Rodrigues do Rego \\ ORCID: https://orcid.org/0000-0003-0891-6438 \\ Universidade Federal do Pará, Brasil \\ E-mail: jr2rego@gmail.com \\ Davi do Socorro Barros Brasil \\ ORCID: https://orcid.org/0000-0002-1461-7306 \\ Universidade Federal do Pará, Brasil \\ E-mail: dsbbrasil18@gmail.com
}

\begin{abstract}
Resumo
A doença de Alzheimer é a perda progressiva da função mental, caracterizada pela degeneração do tecido do cérebro, incluindo a perda de células nervosas, a acumulação de uma proteína anormal chamada $\beta$-amiloide e o desenvolvimento de tranças neuro fibrilares. A agregação de $\beta$-amiloide é uma característica fisiológica da doença de Alzheimer e está intimamente relacionada a neurodegeneração causada pelo Alzheimer. Recentemente novas terapias buscam compostos promissores capazes de desagregar a $\beta$-amiloide. Nanopartículas (NPs) são candidatos altamente promissores para várias aplicações biológicas importantes, como entrega de genes, imagens celulares e terapia de tumores. Entre numerosos tipos de nanomateriais, as NPs de carbono têm atraído interesses particulares, como: fulereno de dimensão zero, nanotubos de carbono unidimensionais (1D) e grafeno bidimensional (2D). Devido a isso, utilizamos molecular docking para investigar a capacidade de interação da nanopartícula de fulereno com o agregado $\beta$-amiloide. Os resultados de docking demonstraram que as principais interações estabelecidas foram hidrofóbicas. $\mathrm{O}$ Sistema teve energia de afinidade de $-41.04 \mathrm{Kcal}$, obtido com o método MM-GBSA.
\end{abstract}

Palavras-chave: Doença de Alzheimer; $\beta$-amiloide; Inibidores; Nanopartícula; Fulereno. 


\begin{abstract}
Alzheimer's disease is the progressive loss of mental function, characterized by degeneration of brain tissue, including the loss of nerve cells, the accumulation of an abnormal protein called $\beta$-amyloid, and the development of neurofibrillary tangles. $\beta$-amyloid aggregation is a physiological feature of Alzheimer's disease and is closely related to neurodegeneration caused by Alzheimer's. Recently, new therapies are looking for promising compounds capable of breaking down $\beta$-amyloid. Nanoparticles (NPs) are highly promising candidates for several important biological applications such as gene delivery, cell imaging and tumor therapy. Among numerous types of nanomaterials, carbon NPs have attracted particular interests, such as: zero-dimensional fullerene, one-dimensional (1D) carbon nanotubes and two-dimensional (2D) graphene. Because of this, we used molecular docking to investigate the interaction capacity of the fullerene nanoparticle with the $\beta$-amyloid aggregate. The docking results showed that the main interactions established were hydrophobic. The System had an affinity energy of -41.04 Kcal, obtained with the MMGBSA method.
\end{abstract}

Keywords: Alzheimer's disease; $\beta$-amyloid; Inhibitors; Nanoparticle; Fullerene.

\title{
Resumen
}

La enfermedad de Alzheimer es la pérdida progresiva de la función mental, caracterizada por la degeneración del tejido cerebral, incluida la pérdida de células nerviosas, la acumulación de una proteína anormal llamada $\beta$-amiloide y el desarrollo de ovillos neurofibrilares. La agregación de $\beta$-amiloide es una característica fisiológica de la enfermedad de Alzheimer y está estrechamente relacionada con la neurodegeneración causada por la enfermedad de Alzheimer. Recientemente, nuevas terapias buscan compuestos prometedores capaces de descomponer el $\beta$-amiloide. Las nanopartículas (NP) son candidatos muy prometedores para varias aplicaciones biológicas importantes, como la administración de genes, la obtención de imágenes celulares y la terapia tumoral. Entre los numerosos tipos de nanomateriales, las NP de carbono han atraído intereses particulares, como: fullereno de dimensión cero, nanotubos de carbono unidimensionales (1D) y grafeno de dos dimensiones (2D). Debido a esto, utilizamos el acoplamiento molecular para investigar la capacidad de interacción de la nanopartícula de fullereno con el agregado $\beta$-amiloide. Los resultados del acoplamiento mostraron que las principales interacciones establecidas fueron hidrofóbicas. El Sistema tuvo una energía de afinidad de $-41.04 \mathrm{Kcal}$, obtenida con el método MM-GBSA.

Palabras clave: Enfermedad de Alzheimer; $\beta$-amiloide; Inhibidores; Nanopartícula; Fullereno.

\section{Introdução}

A doença de Alzheimer (DA) é um distúrbio neurodegenerativo progressivo que destrói gradualmente a função cognitiva do paciente e eventualmente causa sua morte. A DA é a forma mais comum de demência senil em idosos, e estima-se que em 2030 mais de 65 milhões de pessoas em todo o mundo será gravemente afetada por esta terrível doença (Coupé et al., 2019). A fisiopatologia da DA se distingue pelo acúmulo de agregados de $\beta$-amiloides (A $\beta$ ) no cérebro dos pacientes. A hipótese amiloide implica que o desdobramento e a agregação de A $\beta$ desempenha um papel importante na ocorrência e no desenvolvimento de DA. A $\beta$ se agrega em vários tipos de oligômeros, protofibrilas, fibrilas e ficam depositados no tecido neural. Embora estudos iniciais tenham se concentrado nas fibrilas amiloides como causa da DA, há evidências crescentes de que os oligômeros $A \beta$ são os principais agentes citotóxicos responsáveis pela DA (Deture \& Dickson, 2019). Portanto, a relação causal entre a morfologia da agregação de $\beta$-amiloides e a DA permanece controversa, o que dificultou bastante o desenvolvimento de inibidores eficazes para o tratamento da AD. No entanto, a conversão de A $\beta$ do seu estado monomérico solúvel em várias morfologias agregadas no cérebro é agora inegável. Portanto, a inibição de agregados de $\mathrm{A} \beta$ e desagregação de fibrilas $\mathrm{A} \beta$ maduras tem sido apontada como uma estratégia terapêutica atraente para tratamento da DA (Wang et al., 2017).

Um progresso considerável já foi feito, e muitos inibidores amiloides diferentes foram descobertos, incluindo pequenas moléculas orgânicas, proteínas, peptídeos e nanopartículas (Frozza et al., 2018). Entre eles, os inibidores de nanopartículas têm despertado interesse especial devido a suas propriedades físico-químicas únicas, como tamanho pequeno, área de superfície grande, fácil funcionalização e modificação de sua superfície, e boa capacidade de penetração hematoencefálica (Khan et al., 2019).

Nanomateriais de carbono, incluindo nanotubos de carbono, $\mathrm{C}_{60}$, fulereno, grafeno, óxido de grafeno e nanopartículas de ouro têm sido extensivamente estudadas por seus efeitos inibitórios na fibrilogênese amiloide. Muitas pesquisas indicam que os NPs podem interferir na formação dessas estruturas amiloides. Estudos experimentais indicam que os diversos efeitos 
dos nanomateriais na formação de $\mathrm{A} \beta$ depende de suas propriedades intrínsecas e de como esses materiais interagem com as proteínas. A catálise do processo pode ocorrer aumentando a concentração local de proteínas e acelerando a taxa de nucleação na superfície da nanopartícula (NP), enquanto uma ligação estreita ou uma grande área de superfície das partículas com os peptídeos pode levar à inibição da agregação. Apesar dessas observações, os processos detalhados subjacentes à associação de nanomateriais e A $\beta$ até o presente momento não está totalmente elucidado (Hu et al., 2016, 2017; Mankar et al., 2011; Mohajeri et al., 2019).

Vários trabalhos tem utilizado abordagens de modelagem molecular para investigar sistemas biológicas (Cascaes et al., 2021; Castro et al., 2021; Costa et al., 2020; da Silva Costa et al., 2020; Lima et al., 2020). Além disso, essas metodologias tem sido utilizadas para investigar a interação do fulereno com enzimas e peptídeos que estão relacionados e diversos tipos de doenças (Ahmed et al., 2013; Benyamini et al., 2006; De Castro Souza \& Da Silva Gonçalvesa, 2019; Yu et al., 2018). Dessa forma, nesse artigo, vamos investigar o modo de interação de NP de fulereno com a $\beta$-amiloide. Além disso, será avaliado o impacto que essas interações irão causar na estrutura tridimensional do agregado de peptídeos

\section{Metodologia}

\subsection{Molecular docking}

A estrutura molecular do Fulereno foi desenhada usando o software GaussView 5.5 (Dennington et al., 2009; Neto et al., 2020; Santana de Oliveira et al., 2021). Utilizamos o método de molecular docking para prever o modo de interação da nanopartícula de fulereno com a $\beta$-amiloide. O docking foi realizado com o software Molegro Virtual Docker (MVD) 5.5 (Cheung et al., 2013; R. A. Costa et al., 2019; Santana de Oliveira et al., 2020) e a estrutura cristalina da $\beta$-amiloide utilizada como alvo pode ser localizada no Protein Data Bank (http://www.rcsb.org) com o ID: $5 \mathrm{kk} 3$ (Colvin et al., 2016).

A função de pontuação MolDock Score (GRID) foi utilizada com Grid resolution de 0.30 Å e raio de $7 \AA$ A englobando toda o peptideo com o centro localizado em X: -28.84, Y: -31.85 e Z: 23.14 com volume de $81.408 \AA^{3}$ e superfície de 308.48 $\AA^{2}$. O algoritmo MolDock SE foi utilizado com número de execuções igual a 10, 1500 interações máximas e o tamanho máximo da população igual a 50. A avaliação máxima de 300 etapas com distância vizinha fator igual a 1 e limite de energia igual a 100 foi usado durante uma simulação de docking molecular.

\subsection{Simulações dinâmica molecular}

As simulações MD foram realizadas com o pacote de software GROMACS 4.6.2, empregando o campo de força gmx (Hess et al., 2008). O conjunto NVT canônico a $300 \mathrm{~K}$ foi usado com condições de contorno periódicas e a temperatura foi mantida constante pelo termostato de Berendsen. As interações eletrostáticas foram calculadas usando o método de Ewald de malha de partículas (Darden et al., 1993). As distâncias de corte para o cálculo das interações de Coulomb e van der Waals foram 1,0 e 1,4 nm, respectivamente. Antes da simulação dinâmica, a energia do sistema completo foi otimizada sem restrições usando o integrador de descida mais íngreme para 5000 passos. O sistema foi então equilibrado por meio de simulações MD de 100 ps a 300 K. Finalmente, uma simulação de 100 ns foi realizada com um intervalo de tempo de 2 fs.

\subsection{Energia de afinidade}

Para estimar a afinidade de ligação ( $\triangle$ Gbind) usamos os métodos de Mecânica Molecular / Área de Superfície Generalizada (MM-GBSA) (Araújo et al., 2020; Leão et al., 2020; Santos et al., 2020). A $\Delta \mathrm{G}_{\text {bind }}$ foi calculado de acordo com as seguintes equações: 


$$
\begin{gathered}
\Delta \mathrm{G}_{\text {bind }}=\Delta \mathrm{G}_{\text {complex }}-\Delta \mathrm{G}_{\text {receptor }}-\Delta \mathrm{G}_{\text {ligand }}(1) \\
\Delta \mathrm{G}_{\text {bind }}=\Delta \mathrm{H}-\mathrm{T} \Delta \mathrm{S} \approx \Delta \mathrm{E}_{\mathrm{MM}}+\Delta \mathrm{G}_{\text {solv }}-\mathrm{T} \Delta \mathrm{S}(2) \\
\Delta \mathrm{E}_{\mathrm{MM}}=\Delta \mathrm{E}_{\text {internal }}+\Delta \mathrm{E}_{\text {ele }}+\Delta \mathrm{E}_{\mathrm{vdW}}(3) \\
\Delta \mathrm{G}_{\text {solv }}=\Delta \mathrm{G}_{\mathrm{GB}}+\Delta \mathrm{G}_{\mathrm{NP}}(4)
\end{gathered}
$$

A energia livre de ligação $\left(\Delta \mathrm{G}_{\text {bind }}\right)$ é a soma da energia de interação da fase gasosa entre proteína-ligante $\left(\Delta \mathrm{E}_{\mathrm{MM}}\right)$, energia livre de dessolvatação $\left(\Delta \mathrm{G}_{\text {solv }}\right)$ e entropia do sistema (-T $\left.\Delta \mathrm{S}\right) . \Delta \mathrm{E}_{\mathrm{MM}}$ é o resultado da soma da energia interna $\left(\Delta \mathrm{E}_{\text {internal }}\right.$, soma das energias de conexão, ângulos e diédricos) contribuições eletrostáticas ( $\Delta$ Eele) e o termo de van der Waals $\left(\Delta \mathrm{E}_{\mathrm{vdW}}\right)$. $\Delta \mathrm{G}_{\text {solv }}$ é a soma das contribuições polares $\left(\Delta \mathrm{G}_{\mathrm{GB}}\right)$ e não polares $\left(\Delta \mathrm{G}_{\mathrm{NP}}\right) . \mathrm{O} \Delta \mathrm{G}_{\mathrm{SA}}$ foi determinado a partir da área de superfície acessível ao solvente (SASA) estimada pelo algoritmo de combinação linear de sobreposições de pares (LCPO).

\section{Resultados e Discussão}

A simulação de docking molecular foi executada para avaliar como o fulereno é capaz de interagir com a $\beta$-amiloide. Na Figura 1A é exibido o local de interação do fulereno com a $\beta$-Amiloide e na Figura 1B é exibido as interações estabelecidas no complexo $\beta$-amiloide-NP.

Figura 1. (A) Local de ligação da fulereno com a $\beta$-amiloide. (b) Interações estabelecidas no sistema biológico.

(A)

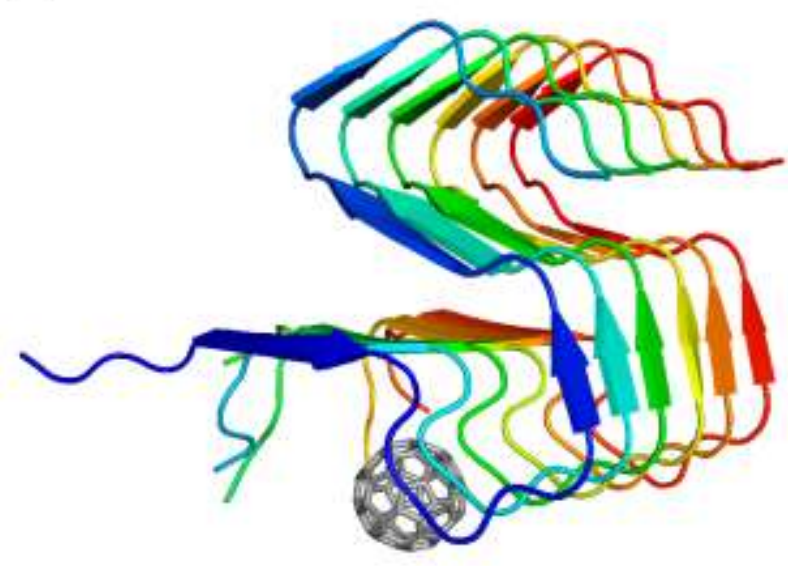

(B)

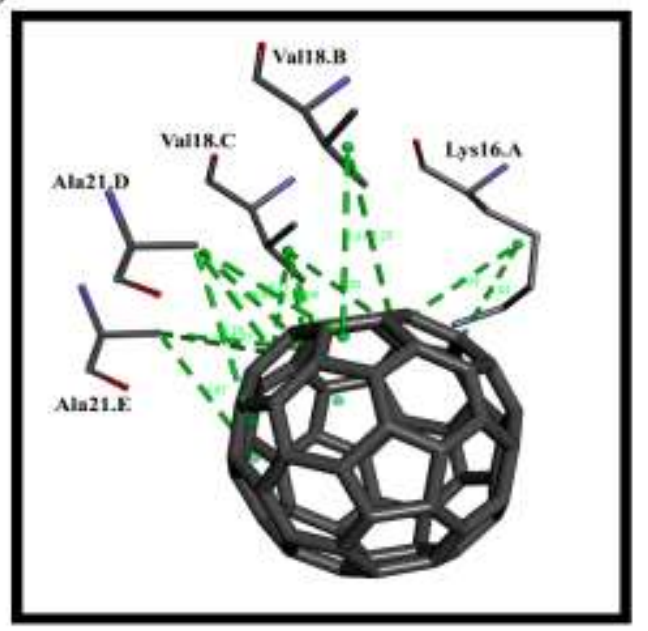

Fonte: Autores.

As interações formadas foram todas de natureza hidrofóbica. Isso ocorre devido a nanopartícula ser formada por basicamente por carbonos, sendo está uma das interações mais comuns formadas por esses átomos. Essas interações foram formadas com resíduos de diferentes cadeias, conforme pode ser visualizado na Figura $2 \mathrm{~B}$. Sendo que essas interações foram com resíduos de Ala21 (pertencente a cadeia E e cadeia D), Val18 (pertencente a cadeia C e cadeia B) e Lys16 da cadeia A.

Simulações de dinâmica molecular foram realizadas para investigar se o complexo formado seria capaz de permanecer interagindo. Após as simulações de dinâmica molecular o método MM-GBSA foi utilizado para calcular a energia de interação do sistema $\left(\Delta \mathrm{G}_{\mathrm{bind}}\right)$. As contribuições energéticas como interações de van der Waals $\left(\Delta \mathrm{E}_{\mathrm{vdw}}\right)$, eletroestáticas $\left(\Delta \mathrm{E}_{\text {ele }}\right)$, polares $\left(\Delta \mathrm{G}_{\mathrm{NP}}\right)$ e não polares $\left(\Delta \mathrm{G}_{\mathrm{N}}\right)$ também foram avaliadas. Os resultados obtidos podem ser visualizados na Tabela 1. 
Tabela 1. Energia de afinidade do sistema $\left(\Delta \mathrm{G}_{\text {bind }}\right)$ e contribuições energéticas obtidas com o método MM-GBSA.

\begin{tabular}{|l|l|l|l|l|l|}
\hline $\mathrm{NP}$ & $\Delta \mathrm{E}_{\mathrm{vdW}}$ & $\Delta \mathrm{E}_{\text {ele }}$ & $\Delta \mathrm{G}_{\mathrm{GB}}$ & $\Delta \mathrm{G}_{\mathrm{NP}}$ & $\Delta \mathrm{G}_{\text {bind }}$ \\
\hline $\mathrm{C}_{60}$ & -43.42 & -6.37 & 14.11 & -5.36 & -41.04 \\
\hline
\end{tabular}

Fonte: Autores.

O resultado de $\Delta \mathrm{G}_{\text {bind }}$ demonstra que a formação do complexo é espontânea. A formação do sistema é conduzida principalmente por interações de van der Waals, seguida pelas interações eletrostáticas e não polares. As contribuições de van der Waals ocorrem principalmente pela natureza química dos átomos envolvidos na interação entre NP e $\beta$-amiloide. Outros trabalhos também evidenciaram que nanopartículas formadas basicamente por carbonos tendem a apresentar $\Delta \mathrm{E}_{\mathrm{vdW}}$ e $\Delta \mathrm{E}_{\mathrm{ele}}$ como as principais contribuições energéticas responsáveis por formar o complexo (Durdagi et al., 2008; Leonis et al., 2015; Qian et al., 2016; Tzoupis et al., 2011). As contribuições da $\Delta \mathrm{E}_{\mathrm{ele}}$ foi um valor abaixo dos resultados de $\Delta \mathrm{E}_{\mathrm{vdw}}$ porque o local de ligação é essencialmente apolar, com poucas cargas eletrostáticas.

\section{Conclusão}

Nesse artigo investigamos a interação do fulereno com a $\beta$-amiloide. Nossos resultados demonstraram que o complexo estabelecido entre a nanopartícula e a $\beta$-Amiloide ocorreu de maneira favorável havendo energia de interação de $41.04 \mathrm{Kcal} / \mathrm{mol}$ calculada utilizando o método MM-GBSA. Nas simulações de docking as interações hidrofóbicas foram as principais responsáveis pela ligação da NP com a $\beta$-amiloide. As interações de van der Waals, eletrostáticas e não polares foram as principais por direcionar a manutenção do sistema formado.

\section{Referências}

Ahmed, L., Rasulev, B., Turabekova, M., Leszczynska, D., \& Leszczynski, J. (2013). Receptor- and ligand-based study of fullerene analogues: Comprehensive computational approach including quantum-chemical, QSAR and molecular docking simulations. Organic and Biomolecular Chemistry, 11(35), 5798-5808. https://doi.org/10.1039/c3ob40878g

Araújo, P. H. F., Ramos, R. S., da Cruz, J. N., Silva, S. G., Ferreira, E. F. B., de Lima, L. R., Macêdo, W. J. C., Espejo-Román, J. M., Campos, J. M., \& Santos, C. B. R. (2020). Identification of potential COX-2 inhibitors for the treatment of inflammatory diseases using molecular modeling approaches. Molecules, 25(18), 4183. https://doi.org/10.3390/molecules25184183

Benyamini, H., Shulman-Peleg, A., Wolfson, H. J., Belgorodsky, B., Fadeev, L., \& Gozin, M. (2006). Interaction of C60-fullerene and carboxyfullerene with proteins: Docking and binding site alignment. Bioconjugate Chemistry, 17(2), 378-386. https://doi.org/10.1021/bc050299g

Cascaes, M. M., Silva, S. G., Cruz, J. N., Santana de Oliveira, M., Oliveira, J., Moraes, A. A. B. de, Costa, F. A. M. da, da Costa, K. S., Diniz do Nascimento, L., \& Helena de Aguiar Andrade, E. (2021). First report on the Annona exsucca DC. Essential oil and in silico identification of potential biological targets of its major compounds. Natural Product Research. https://doi.org/10.1080/14786419.2021.1893724

Castro, A. L. G., Cruz, J. N., Sodré, D. F., Correa-Barbosa, J., Azonsivo, R., de Oliveira, M. S., de Sousa Siqueira, J. E., da Rocha Galucio, N. C., de Oliveira Bahia, M., Burbano, R. M. R., do Rosário Marinho, A. M., Percário, S., Dolabela, M. F., \& Vale, V. V. (2021). Evaluation of the genotoxicity and mutagenicity of isoeleutherin and eleutherin isolated from Eleutherine plicata herb. using bioassays and in silico approaches. Arabian Journal of Chemistry, 14(4), 103084. https://doi.org/10.1016/j.arabjc.2021.103084

Cheung, J., Gary, E. N., Shiomi, K., \& Rosenberry, T. L. (2013). Structures of human acetylcholinesterase bound to dihydrotanshinone i and territrem B show peripheral site flexibility. ACS Medicinal Chemistry Letters, 4(11), 1091-1096. https://doi.org/10.1021/ml400304w

Colvin, M. T., Silvers, R., Ni, Q. Z., Can, T. V., Sergeyev, I., Rosay, M., Donovan, K. J., Michael, B., Wall, J., Linse, S., \& Griffin, R. G. (2016). Atomic Resolution Structure of Monomorphic A 342 Amyloid Fibrils. Journal of the American Chemical Society, 138(30), 9663-9674. https://doi.org/10.1021/jacs.6b05129

Costa, E. B. B., Silva, R. C. C., Espejo-Román, J. M. M., Neto, M. F. de A. F. d. A., Cruz, J. N. N., Leite, F. H. A. H. A., Silva, C. H. T. P. H. T. P., Pinheiro, J. C. C., Macêdo, W. J. C. J. C., \& Santos, C. B. R. B. R. (2020). Chemometric methods in antimalarial drug design from 1,2,4,5-tetraoxanes analogues. SAR and QSAR in Environmental Research, 31(9), 1-19. https://doi.org/10.1080/1062936X.2020.1803961

Costa, R. A., Cruz, J. N., Nascimento, F. C. A., Silva, S. O. S. G., Silva, S. O. S. G., Martelli, M. C., Carvalho, S. M. L., Santos, C. B. R., Neto, A. M. J. C., \& Brasil, D. S. B. (2019). Studies of NMR, molecular docking, and molecular dynamics simulation of new promising inhibitors of cruzaine from the parasite Trypanosoma cruzi. Medicinal Chemistry Research, 28(3), 246-259. https://doi.org/10.1007/s00044-018-2280-z

Coupé, P., Manjón, J. V., Lanuza, E., \& Catheline, G. (2019). Lifespan Changes of the Human Brain In Alzheimer's Disease. Scientific Reports, 9(1), 1-12. https://doi.org/10.1038/s41598-019-39809-8 
da Silva Costa, A. C., Carvalho, S. C., de Farias Silva, N., do Nascimento-Júnior, A. E. S., Cruz, J. N., de Jesus Chaves Neto, A. M., do Socorro Barros Brasil, D., Silva-Júnior, J. O. C., \& Ribeiro-Costa, R. M. (2020). Effect of chitosan/albendazole nanocarriers' solvation by molecular dynamics. Theoretical Chemistry Accounts, 139(7), 1-15. https://doi.org/10.1007/s00214-020-02620-0

Darden, T., York, D., \& Pedersen, L. (1993). Particle mesh Ewald: An N· $\log (\mathrm{N})$ method for Ewald sums in large systems. The Journal of Chemical Physics, 98(12), 10089-10092. https://doi.org/10.1063/1.464397

De Castro Souza, I., \& Da Silva Gonçalvesa, A. (2019). Computational studies of fullerene derivatives as pesticide captors. Orbital, 11(1), 10-17. https://doi.org/10.17807/orbital.v11i1.1215

Dennington, R., Keith, T., \& Millam, J. (2009). GaussView, Version 5. In Semichem Inc. , Shawnee Mission, KS (p. Semichem Inc).

Deture, M. A., \& Dickson, D. W. (2019). The neuropathological diagnosis of Alzheimer's disease. In Molecular Neurodegeneration (Vol. 14, Issue 1, pp. 118). BioMed Central Ltd. https://doi.org/10.1186/s13024-019-0333-5

Durdagi, S., Mavromoustakos, T., Chronakis, N., \& Papadopoulos, M. G. (2008). Computational design of novel fullerene analogues as potential HIV-1 PR inhibitors: Analysis of the binding interactions between fullerene inhibitors and HIV-1 PR residues using 3D QSAR, molecular docking and molecular dynamics simulations. Bioorganic and Medicinal Chemistry, 16(23), 9957-9974. https://doi.org/10.1016/j.bmc.2008.10.039

Frozza, R. L., Lourenco, M. V., \& de Felice, F. G. (2018). Challenges for Alzheimer's disease therapy: Insights from novel mechanisms beyond memory defects. In Frontiers in Neuroscience (Vol. 12, Issue FEB, p. 37). Frontiers Media S.A. https://doi.org/10.3389/fnins.2018.00037

Hess, B., Kutzner, C., Van Der Spoel, D., \& Lindahl, E. (2008). GRGMACS 4: Algorithms for highly efficient, load-balanced, and scalable molecular simulation. Journal of Chemical Theory and Computation, 4(3), 435-447. https://doi.org/10.1021/ct700301q

Hu, T., Chen, C., Huang, G., \& Yang, X. (2016). Antibody modified-silver nanoparticles for colorimetric immuno sensing of A $\beta(1-40 / 1-42)$ based on the interaction between $\beta$-amyloid and Cu2+. Sensors and Actuators, B: Chemical, 234, 63-69. https://doi.org/10.1016/j.snb.2016.04.159

Hu, T., Lu, S., Chen, C., Sun, J., \& Yang, X. (2017). Colorimetric sandwich immunosensor for A $\beta(1-42)$ based on dual antibody-modified gold nanoparticles. Sensors and Actuators, B: Chemical, 243, 792-799. https://doi.org/10.1016/j.snb.2016.12.052

Khan, I., Saeed, K., \& Khan, I. (2019). Nanoparticles: Properties, applications and toxicities. In Arabian Journal of Chemistry (Vol. 12, Issue 7, pp. 908-931). Elsevier B.V. https://doi.org/10.1016/j.arabjc.2017.05.011

Leão, R. P., Cruz, J. V. J. N., da Costa, G. V., Cruz, J. V. J. N., Ferreira, E. F. B., Silva, R. C., de Lima, L. R., Borges, R. S., Dos Santos, G. B., \& Santos, C. B. R. (2020). Identification of new rofecoxib-based cyclooxygenase-2 inhibitors: A bioinformatics approach. Pharmaceuticals, 13(9), 1-26. https://doi.org/10.3390/ph13090209

Leonis, G., Avramopoulos, A., Papavasileiou, K. D., Reis, H., Steinbrecher, T., \& Papadopoulos, M. G. (2015). A Comprehensive Computational Study of the Interaction between Human Serum Albumin and Fullerenes. Journal of Physical Chemistry B, 119(48), 14971-14985. https://doi.org/10.1021/acs.jpcb.5b05998

Lima, A. R. J. A. de M., Siqueira, A. S., Möller, M. L. S., Souza, R. C. de, Cruz, J. N., Lima, A. R. J. A. de M., Silva, R. C. da, Aguiar, D. C. F., Junior, J. L. da S. G. V., \& Gonçalves, E. C. (2020). In silico improvement of the cyanobacterial lectin microvirin and mannose interaction. Journal of Biomolecular Structure and Dynamics. https://doi.org/10.1080/07391102.2020.1821782

Mankar, S., Anoop, A., Sen, S., \& Maji, S. K. (2011). Nanomaterials: amyloids reflect their brighter side. Nano Reviews, 2(1), 6032. https://doi.org/10.3402/nano.v2i0.6032

Mohajeri, M., Behnam, B., Barreto, G. E., \& Sahebkar, A. (2019). Carbon nanomaterials and amyloid-beta interactions: potentials for the detection and treatment of Alzheimer's disease? In Pharmacological Research (Vol. 143, pp. 186-203). Academic Press. https://doi.org/10.1016/j.phrs.2019.03.023

Neto, R. de A. M. M., Santos, C. B. R. R., Henriques, S. V. C. C., Machado, L. de O., Cruz, J. N., da Silva, C. H. T. d. P. T. de P., Federico, L. B., Oliveira, E. H. C. d. C. de, de Souza, M. P. C. C., da Silva, P. N. B. B., Taft, C. A., Ferreira, I. M., \& Gomes, M. R. F. F. (2020). Novel chalcones derivatives with potential antineoplastic activity investigated by docking and molecular dynamics simulations. Journal of Biomolecular Structure and Dynamics, 1-13. https://doi.org/10.1080/07391102.2020.1839562

Qian, M., Shan, Y., Guan, S., Zhang, H., Wang, S., \& Han, W. (2016). Structural Basis of Fullerene Derivatives as Novel Potent Inhibitors of Protein Tyrosine Phosphatase 1B: Insight into the Inhibitory Mechanism through Molecular Modeling Studies. Journal of Chemical Information and Modeling, 56(10), 20242034. https://doi.org/10.1021/acs.jcim.6b00482

Santana de Oliveira, M., Neves da Cruz, J., Almeida da Costa, W., Silva, S. G., da Paz Brito, M., Fernandes de Menezes, S. A., de Jesus Chaves Neto, A. M., de Aguiar Andrade, E. H., de Carvalho, R. N., da Cruz, J. N., Almeida da Costa, W., Silva, S. G., Brito, M. da P., de Menezes, S. A. F., de Jesus Chaves Neto, A. M., de Aguiar Andrade, E. H., de Carvalho Junior, R. N., Oliveira, M. S. De, Neves, J., \& de Carvalho, R. N. (2020). Chemical Composition, Antimicrobial Properties of Siparuna guianensis Essential Oil and a Molecular Docking and Dynamics Molecular Study of its Major Chemical Constituent. Molecules, 25(17), 3852. https://doi.org/Manuscript accepted

Santana de Oliveira, M., Pereira da Silva, V. M., Cantão Freitas, L., Gomes Silva, S., Nevez Cruz, J., \& de Aguiar Andrade, E. H. (2021). Extraction Yield, Chemical Composition, Preliminary Toxicity of Bignonia nocturna (Bignoniaceae) Essential Oil and in Silico Evaluation of the Interaction. Chemistry and Biodiversity, cbdv.202000982. https://doi.org/10.1002/cbdv.202000982

Santos, C. B. R., Santos, K. L. B., Cruz, J. N., Leite, F. H. A., Borges, R. S., Taft, C. A., Campos, J. M., \& Silva, C. H. T. P. (2020). Molecular modeling approaches of selective adenosine receptor type 2A agonists as potential anti-inflammatory drugs. Journal of Biomolecular Structure and Dynamics. https://doi.org/10.1080/07391102.2020.1761878

Tzoupis, H., Leonis, G., Durdagi, S., Mouchlis, V., Mavromoustakos, T., \& Papadopoulos, M. G. (2011). Binding of novel fullerene inhibitors to HIV-1 
Research, Society and Development, v. 10, n. 11, e261101119585, 2021

(CC BY 4.0) | ISSN 2525-3409 | DOI: http://dx.doi.org/10.33448/rsd-v10i11.19585

protease: Insight through molecular dynamics and molecular mechanics Poisson-Boltzmann surface area calculations. Journal of Computer-Aided Molecular Design, 25(10), 959-976. https://doi.org/10.1007/s10822-011-9475-4

Wang, J., Gu, B. J., Masters, C. L., \& Wang, Y. J. (2017). A systemic view of Alzheimer disease - Insights from amyloid- $\beta$ metabolism beyond the brain. In Nature Reviews Neurology (Vol. 13, Issue 10, pp. 612-623). Nature Publishing Group. https://doi.org/10.1038/nrneurol.2017.111

Yu, Y., Sun, H., Hou, T., Wang, S., \& Li, Y. (2018). Fullerene derivatives act as inhibitors of leukocyte common antigen based on molecular dynamics simulations. RSC Advances, 8(25), 13997-14008. https://doi.org/10.1039/c7ra13543b 